\title{
Le Rhône et son Utilisation ${ }^{1)}$
}

\author{
Par M. DENIZET, Inspecteur Général honoraire des Ponts et Chaussées.
}

La loi du 27 mai 1921 a décidé en principe l'aménagement intégral du Rhône au triple point de vue de la navigation, des forces motrices et de l'irrigation, et a décidé en même temps, pour la réalisation de cet aménagement, la création d'une grande Société nationale disposant d'un capital actions et obligations de 3 milliards de francs et composée de toutes les grandes collectivités et de toutes les grandes industries de la région intéressée.

Cette décision met fin législativement à une controverse très ancienne et donne satisfaction, au moins en théorie, à tous les desiderata que le Rhône avait fait naître. La formule de l'aménagement intégral satisfait complètement notre esprit de logique et notre tendance à la symétrie, en même temps que le groupement de tous les intéressés dans la grande société chargée de tirer le meilleur parti du Rhône résout par avance toutes ies compétitions et toutes les jalousies qui commençaient à se faire jour. Mais sera-t-il possible de réaliser pratiquement dans son intégralité le beau programme qu'on s'est donné et qui n'est encore appuyé que sur des études sommaires? Qu'on permette à un ancien Ingénieur du Rhône de présenter quelques observations à ce sujet.

J'examinerai successivement la question pour le Bas-Rhône et pour le Haut-Rhône car ces deux parties du fleuve sont essentiellement différentes, tant au point de vue technique qu'au point de vue économique et il n'est pas possible d'arriver à des conclusions communes pour ces deux sections.

\section{BAS-RHONE}

Navigation. - Le Bas-Rhône a été l'objet de travaux de régularisation s'étendant sur tout son parcours, exécutés en vertu de la loi du 13 mai 1878 et aujourd'hui terminés. Le résultat a été à peu près celui qu'on s'était proposé. Le tirant d'eau minimum qui descendait, avant les travaux jusqu'à $0 \mathrm{~m}$. 40 ne descend plus aujourd'hui au-dessous de $1 \mathrm{~m}$. 40 et sept hauts-fonds seulement présentent ce minimum. Le tirant d'eau de $1 \mathrm{~m}$. 60 qu'on cherchait à réaliser en tous temps est assuré pendant 330 jours par an. Ces résultats s'entendent pour une année normale ; ils ne s'appliquent pas à l'année 1921 qui a été absolument exceptionnelle et qui a été désastreuse pour la navigation sur tous les cours d'eau simplement.régularisés. Grâce à ces résultats, la navigation s'est notablement développée sur le Bas-Rhône entre Lyon et Arles. Deux grandes sociétés y font circuler un matériel nombreux et auraient déjà pris, sans la guerre, un grand développement.

On voudrait faire mieux et l'un des articles du programme d'aménagement consisterait à donner au Rhône des conditions de navigabilité comparables à celles de la Seine et de la Saône et du canal de Bourgogne qui les réunit : 2 mètres de tirant d'eau minimum en tous temps et une vitesse réduite permettant une remonte facile. Comment peut-on arriver à ce résultat?

Par la régularisation, on ne peut songer à obtenir sensiblement plus que ce qu'on a obtenu jusqu'à présent. Les résultats auxquels on est arrivé sur le Rhin sont beaucoup plus satisfaisants et plus complets. Mais aussi, il y a entre le Rhin et le Rhône des différences profondes qu'il ne faut pas perdre de vue.

Le Rhin à Strasbourg et le Rhône à Lyon, à son confluent avec la Saône, ont a peu près la même pente et la même vitesse. Mais le Rhin n'est qu'à la cote 134.80 à l'étiage et a encore $734 \mathrm{~km}$. à parcourir pour arriver à la mer du Nord, tandis que le Rhône est à la cote 157,67 et n'est séparé de la Méditerranée que par un parcours de $335 \mathrm{~km}$.

Le Rhône a done une pente moyenne plus que double de celle du Rhin. Mais, en outre, celui-ci va constamment en s'améliorant de Strasbourg à la mer, la courte section de Bingen à Saint-Goar mise à part, tandis que le Rhône s'aggrave notablement sur les $90 \mathrm{~km}$. qui s'étendent entre le confluent de l'Isère et celui de l'Ardèche, section où la pente moyenne atteint $0 \mathrm{~m}$. 77 par $\mathrm{km}$. et où la pente maxima est triple ou quadruple ; on verra plus loin comment cette aggravation s'explique par les nombreux torrents qui tombent dans le Rhône sur l'étendue de cette section. Enfin, si on ajoute que le débit du Rhin est en eaux basses et en eaux moyennes d'un quart supérieur à celui du Rhône, on ne peut s'étonner que le premier fleuve présente, avec le même système de travaux, des conditions de navigabilité autrement meilleures que le second. Il serait chimérique de vouloir prendre les résultats obtenus sur le Rhin comme but à atteindre pour le Rhòne et on doit se borner à perfectionner ce que l'on a réalisé, sans espoir d'en changer notablement les traits essentiels.

Un moyen radical d'améliorer la navigation entre Lyon et Arles consisterait à créer un canal latéral sur toute cette longueur. On avait envisagé cette solution en 1900 et on avait trouvé que. pour un canal de navigation seulement, sans utilisation des forces motrices, c'est-à-dire à faible débit et à section modérée, la dépense serait d'environ 600 millions ; il faudrait aujourd'hui envisager une dépense de deux milliards. C'est dire qu'une telle solution est inapplicable.

Il ne reste alors que la solution consistant à faire des dérivations éclusées sur les points difficiles. Mais on ne peut songer à le faire qu'au droit des principaux hauts-fonts. La plus grande partie du cours du fleuve restera libre et par conséquent l'obstacle de la vitesse, qui est le principal, ne sera pas écarté.

D'autre part, les dérivations devront être accompagnées ì leur tête d'un barrage en travers du Rhône car on ne peut concevoir l'alimentation pratique de dérivation sur un cours d'eall à fond mobile, comme le Rhône, par de simples coupures de la berge Or, si peu élevés que soient ces barrages et si mobiles qu'ils soient en cas de crues ils apporteront cependant au régime actuel du fleuve des modifications qui pourront avoir des répercusssions graves à l'amont. C'est un point que j'examinerai tout à l'heure plus à fond à propos de l'utilisation des forces motrices. Il suffit de dire ici qu'il ne faudra procéder qu'avec la plus grande prudence de facon à ne pas compromettre en vue d'amélioration d'un intérêt pratique modeste, les résultats très importants que l'on a déjà acquis par la simple régularisation. Dans ces limites et avec cette façon de faire, on peut vraisemblablement améliorer encore un peu les conditions actuelles de navigabilité du Rhône. Mais ces travaux coûteront cher pour un résultat médiocre et c'est ainsi qu'on est amené à se demander si on ne peut combiner les deux parties essentielles de l'aménagement, c'est-à-dire la navigation et les forces motrices, de façon à faire payer par cellesci au moins la plus grande partie des dépenses de celle-là.

Utilisation des forces motrices. - Le Bas-Rhône n'est encore, à l'heure actuelle, utilisé sur aucun point pour la force motrice. Comme il peut fournir, d'après les estimations les plus modestes, environ $350.000 \mathrm{kw}$, il est naturel qu'on ait songé à tirer part de cette puissance jusqu'ici inutilisée. Et comme la navigation se trouve déjà installée, on a cherché le moyen de la conserver et même de l'améliorer tout en réalisant l'utilisation des forces motrices. Mais on se heurte tout de suite dans cet ordre d'idées à de graves difficultés.

Faire un canal latéral servant à la fois à la navigation et aux forces motrices, comme le canal projeté d'Alsace entre Bâle et Strasbourg, est impossible si on considère que la dépense indiquée plus haut de deux milliards pour un canal de navigation seulement devrait être considérablement majorée pour tenir compte de l'augmentation du débit à dériver et de l'accroissement de la section nécessaire pour maintenir dans le canal une vitesse compatible avec une bonne navigation.

La chose est possible en Alsace où les conditions du terrain sont favorables; elle ne le serait pas dans la vallée du Rhone ol le canal rencontrerait à chaque pas des obstacles insurmontables ou exigeant, pour être surmontés, d'énormes dépenses. On avait prévu primilivement pour le canal d'Alsace une vitesse de $1 \mathrm{~m} .20$ par scconde que l'on considérait comme admissible pour la remonte des bateaux. Les représentants de la navigation demandent qu'on 
ne dépasse pas $0 \mathrm{~m}$. 70. Que l'on' admette l'un ou l'autre chiffre pour un canal latéral au Rhône, c'est toujours une section énorme qu'on ne voil pas la possibilité de loger pratiquement et sans dépenses excessives sur l'une ou l'autre rive du fleuve.

Mais si un canal lateral de bout en bout est impossible, ne peut on obtenir à peu près le même résultat au moyen de dérivations assez nombreuses? Au droit des passages difficiles du fleuve et toutes les fois que les conditions du terrain le permettraient, on établirait une section de canal latéral qui aurait le double avantage de contourner le passage difficile et de créer une puissance hydraulique dont l'importance augmenterait avec la longueur sur laquelle pourrait se développer la dérivation. Celle-ci serait accompagnée en tête d'un barrage en travers du Rhône de faible hauteur ; elle aurait une section suffisante pour véhiculer le volume nécessaire à la mise en jeu de l'usine hydro-électrique sans présenter une vitesse gênante pour la navigation ; enfin elle rentrerail dans le fleuve par une écluse accompagnée de l'usine hydroelectrique.

Tel est l'ordre d'idées dans lequel l'Administration s'est placée en étudiant sommairement l'utilisation du Bas-Rhône et telle est également la conception sur lacquelle travaillent les demandeurs eventuels en concession. On ne voit d'ailleurs pas la possibilité de faire mieux. Malheureusement cette conception ellemême soulève de graves objections.

En premier lieu, les diverses dérivations n'occuperont certainement pas tout le cours du Rhône. Il faudra donc encore utiliser celui-ci dans son état naturel régularisé, c'est-à-dire avec ses fortes pentes et ses grandes vitesses, et on n'évitera pas l'usage d'un matériel spécial pour la navigation du Rhône, ce qui serait pourtant si désirable.

Fin second lieu, et c'est là l'objection essentielle, il est fort à craindre que les barrages à établir en tête des dérivations n'amènent des perturbations profondes dans les parties du fleuve situées à l'amont de ces barrages. Je n'ignore pas que ceux-ci seront parfaitement mobiles et susceptibles d'être manœuvrés rapidement de façon à rétablir à volonté en très peu de temps l'écoulement naturel du fleuve. Mais il n'en est pas moins vrai qu'ils resteront fermćs pendant les basses eaux de facon à assurer l'alimentation des dérivations et des usines hydro-électriques.

Or, le Rhône charrie en tout temps du gravier. Par les plus basses eaux on entend en passant en batelet sur les parties où la profondeur est faible, le bruit des galets qui roulent sur le fond. Que deviendront ces graviers pendant la fermeture des barrages? En outre ceux-ci ne pourront pas être ouverts dès que le débit aura dépassé celui d'étiage, ni être fermés seulement lorsqu'on sera descendu exactement à ce même débit d'étiage. Les barrages seront fermés, au moins en partie pendant un certain temps audelà du dépassement de l'étiage et avant le retour à celui-ci. Pendant les ouvertures partielles l'écoulement se fera à travers le barrage, suivant une section différente de celle qui existerait si le barrage n'existait pas. Enfin, il ne peut être contesté que, même en cas d'ouverture totale, le débit du fleuve sera diminué à l'aval de tout le volume introduit dans la dérivation. L'équilibre réalisé avec tant de peines au moyen de la régularisation se trouvera donc troublé et, s'il est impossible de dire exactement quelles seront les conséquences de ce trouble, on ne peut pas ne pas être inquiet sur la nature et la portée de ces conséquences et sur le sort final qui serait réservé, dans ces conditions, à la navigation du Rhône.

Il faut dire que cette question du transport des graviers a, sur le Rhône, une importance que l'on ne rencontre pas ailleurs. En amont de Lyon et en aval du Lac Léman, le Rhône recoit déjà plusieurs affluents torrentiels dont les apports sont considérables, notamment l'Arve et l'Ain. Mais c'est surtout en aval de Lyon que les circonstances sont exceptionnellement défavorables. La le Rhône coule sur presque toute sa longueur dans un couloir bordé de montagnes assez élevées d'où se précipitent un grand nombre de torrents qui lui apportent au moment de leurs crues des volumes relativement énormes et des paquets de graviers qui sont certainement en rapport avec ces volumes. Ces affluents, dont quelques-uns sont presque à sec ou même complètement à sec en été, jettent dans le fleuve, au moment des crues, des débits comparables à ceux des grands affluents venant des Alpes : l'Isère et la Durance ; c'est" le cas, par exemple, du Doux, de l'Eyrieux, de l'Escoutaille, de l'Ardèche, du Gardon sur la rive droite, du Roubion, de l'Eygues, du Lez sur la rive gauche. On comprend combien les apports subits et considérables de ces torrents rendent difficile la stabilisation du régime du Rhône et combien ils doi. vent imposer de circonspection lorsqu'il s'agit de toucher à ce régime.

On peut donc se demander s'il est pratiquement possible de combiner les deux points de vue navigation et forces motrices et si l'on ne se verra pas dans l'obligation de sacrifier l'un ou l'autre. Quel serait, dans ce cas, celui qui devrait être abandonné? La navigation a pour elle en quelque sorte sa longue possession et les grands services qu'elle a rendus au commerce et à l'activité économique de la région. Avant la guerre, on la considérait comme un frein utile contre l'élévation des tarifs de chemins de fer. Aujourd'hui que les grandes compagnies ne sont plus guère que des gérants de l'Etat et que, par suite, la concurrence entre la voie d'eau et la voie ferrée a perdu beaucoup de son acuité, ce point de vue n'a plus la même importance ; mais le Rhône restera toujours, sinon comme un rival, tout au moins comme un auxiliaire extrêmement utile du chemin de fer, et sa disparition ou sa déchéance comme voie navigable aurait les plus fâcheuses conséquences. Il en est tout autrement des forces motrices. Sans doute celles-ci sont des plus intéressantes par leur importance et par leur situation. Mais on peut trouver l'équivalent et à meilleur compte sur les affluents dont beaucoup, malgré leurs conditions avantageuses, ne sont pas encore ou commencent à peine à être utilisés. La question des forces motrices est donc beaucoup moins vitale pour le Bas-Rhône que celles de la navigation et, à mon avis, c'est celle-ci qui doit l'emporter, c'est-à-dire qu'on ne doit rien faire qui puisse la compromettre et que, si l'on veut s'engager dans la voie de l'aménagement combiné, ce doit être avec une extrême prudence et à condition de pouvoir s'appuyer sur des exemples d'autres cours d'eal où une telle combinaison ait été réalisée avec succès.

Irrigations. - J'ai laissé de côté intentionnellement les irrigations parce que c'est une question qui pourrait être, dans tous les cas, facilement résolue dans la mesure où l'irrigation serait justifiée.

La vallée du Rhône proprement dite n'offre qu'une surface relativement faible et on trouvera toujours dans le fleuve, sans gêner aucune autre utilisation, l'eau nécessaire pour l'irrigation de cette surface. Il n'y a pas seulement, il est vrai, que la vallée du Rhône à considérer, on a envisagé l'irrigation d'une partie des plateaux qui la bordent. Mais, dans cette voie, il est facile de se rendre compte qu'on ne peut aller pratiquement bien loin.

Pour élever un litre d'eau à 30 mètres, il faut dépenser en travail utile $30 \mathrm{kgm}$. ou $50 \mathrm{kgm}$. brut, c'est-à-dire que pour élever à cette hauteur de 30 mètres continu de 1 litre par seconde, un débit correspondant à l'irrigation d'un hectare, il faut une puissance d'environ $1 / 2 \mathrm{kw}$. Si on admet 4.000 heures pour la durée annuelle des irrigations, il faut donc fournir en force motrice pour l'arrosage d'un hectare à cette hauteur environ $2.000 \mathrm{kwh}$. D'après des estimations qui ont été faites pour des usines projetées sur des affluents du Bas-Rhône, on ne peut évaluer le prix de revient du kwh. transporté à l'usine élévatoire à moins de $0 \mathrm{fr} .10$, ce qui fait une dépense annuelle d'énergie électrique par hect. de 200 francs, chiffre au-delà duquel il parait difficile d'aller, car, en ajoutant environ 100 francs pour charges de premier établissement et entretien du réseau de distribution, ainsi que pour frais d'administration, on arrive à un total de 300 francs qui peut être-considéré comme la limite admissible de ce qu'on peut dépenser pour l'irrigation d'un hectare. Les surfaces à arroser en dehors de la vallée même du Rhône seront donc peu étendues et l'énergie nécessaire à leur irrigation sera, dans tous les cas, facile à trouver.

\section{Haut-Rhone.}

La situation sur le Haut-Rhône est toute différente de celle du Bas-Rhône.

Navigation. - Il n'a été encore presque rien fait pour la navigation sur le Haut-Rhône français. En dehors de quelques rar es ouvrages de régularisation, il $n^{\prime} y$ a à citer que la dérivation écl ${ }^{l}$ sée du Sault et celle de Jonage immédiatement en amont de Lyon dérivation faite pour l'usine hydro-électrique de ce nom et accompagnée des écluses nécessaires au maintien de la navigation. Celle-ci est d'ailleurs très peu active depuis son origine officielle au Parc, à la sortie des gorges de Bellegarde jusqu'aux abords de Lyon où elle prend quelque importance pour le transpor $t$ de matériaux de construction. 
On pourrail sans aucun donte améliorer cete situation si la question présentait un intérêt sulfisant, c'est-à-dire si l'on pouvait espérer un trafic important. Nais pour cela il faut, de toute nécessité, aller jusqu'au lac I cimail : aucune discussion n'est, je crois, possible sur ce point. Or, de Lyon au lac téman, le Rhòne présente trois sections totalement différentes. La première, de Lyon jusquau Pare, à la sortie des gorges de Bellegarde, est assimilable comme pente à la partic du Bas-Rhòne situe entre Lyon et le confluent de l'Isère, mais avec un débit beatucoup moindre et ne pourrait, par consécuent, que difficilement être améliorće par la régularisation. La seconde, formée par les gorges de Bellegarde (traversée du Jura), ne peut etre rendue navigable que par des travaux grandioses que la navigation seule ne pourrait justifier et qui ne peurent être entrepris qu'en rue de laménagement des forces motrices considérables que l'on projette depuis longtemps de tirer de cette section. Enfin, la troisième partie. comprise entre les gorges et le lad Léman, n'a ćté encore elle, non plus, l'objet d'aucun travail de navigation et l'usine de forces motrices de Chèvres créée sur le territoire suisse n'est pas accompagnce d'écluses.

Il faut dire cependant que l'usine intermationale de PougnySancy, en construction à l'aval de Chèves entre les territoires français et suisse, est projetée avec une écluse de $80 \times 12 \times 2,50$ mais dont la tête amont senlement doit être exécutće pour le moment. En outre, l'arrivée dans le lac Léman serait particulièrement difficile.

En résumé, tout ou presque tout est à faire sur le Haul-Rhòne au point de vue navigation et les problèmes qui se présentent à. ce point de vue sont tellement complexes et onćreux qu'on né peut pratiquement songer à les résoudre sans les combiner avec l'utilisation des forces motrices.

Forces motrices. - Comme forces motrices, le Haut-Rhòne n'est encore utilisé qu'à Jonage et sur le territoire suisse. Il offre encore à cet égard de très grandes disponibilités, qui peuvent être évaluées pour le territoire français à $350.000 \mathrm{kw}$. c'est-à-dire sensiblement au même chiffre que ce que peut offrir le Bas-Rhòne, une grande partie de cette puissance se trouvant d'ailleurs accumulée dans les gorges et étant par conséquent d'un aménagement très avantageux. Mais si on veut combiner cet aménagement des forces motrices du Haut-Rhône avec les exigences de la navigation, ne se trouvera-t-on pas en présence des mêmes difficultés que celles qui ont été signalées pour le Bás-Rhône? Il est clair, par exemple, qu'on rencontrera clans la première section, entre Lyon et les gorges, ainsi que dans la troisième, entre les gorges et le confluent de l'Arve, les difficultés provenant du transport des graviers. Peut-être ce transport sera-t-il moins redoutable en raison de la décantation, par le lac Léman, đu Rhône lui-même. Les résultats obtenus dans le remous des usines établies sur le territoire suisse, donneront sans doute quelques renseignements à ce sujet, mais en escomptant un peu plus de facilités à cet égard sur le Haut-Rhône que sur le Bas-Rhône, il ne faut pas se dissimuler que, sur le premier comme sur le second, il sera probablement très difficile sinon impossible de combiner les exigences d'une bonne navigation avec l'utilisation avantatageuse des forces motrices, et ici, comme pour le Bas-Rhône, la question se pose : quel est le point de vue qui doit céder devant l'autre?

Mais ici, la réponse sera différente. La navigation n'existe que peu ou point sur le Haut-Rhône. Pour la créer, il faudrait aller jusqu'au lac Léman et dépenser des sommes qui, au point de vue économique tout au moins, paraissent hors de proportion avec Ie résultat à atteindre. Au contraire, les forces motrices pourraient être aménagées dans des conditions avantageuses et fournir à l'industrie française, même à une grande distance du lieu de production des quantités d'énergie à bon marché consiclérables. Il semble donc qu'on ne doive conclure à l'abandon du point de vue navigation et à la réalisation immédiate des forces motrices.

Il ne conviendrait pas cepèndant de trancher aussi vite une question qui, même au point de vue économique, mais surtout au point de vue politique, est des plus complexes et des plus délicates. Au point de vue économique, ce n'est pas seulement la Suisse romande qu'on pourrait desservir en canalisant le Haut-Rhône, mais encore la Suisse septentrionale, grâce au canal d'Entreroches que nos voisins d'au delà du Jura songent à établir entre la lac Léman et le Rhin à Bâle par les lacs de Neuchâtel et de
Bienne et la rivière d'Aar. Au poinl de vue politique, on sait qu'un traite de 1798 avait été conclu entre la France et la Suisse pour la réunion des deux pays par un canal longeant ou empruntant le Rhòne et nous aurions peul-être mauvaise gràce à ne pas nous prêter à l'exécution de cet accord si nos voisins se montraient de leur coté disposés à en poursuivre la réalisation, d'autant plus que l'entente à ce sujet entrainerait naturellement l'accord relatif à la bonne utilisation du lac Léman pour les usines de forces motrices d'aval.

Toulefois, le point de vue qui vient d'être exposé n'est pas aussi simple qu'on pourrait le croire au premier abord. Le Rhône communique deja avec le Rhin à Bàle par le canal du Rhône au Rhin qu'on travaille à mettre au gabaril de la péniche.

Il n'est pas impossible qu'on aille plus loin. Avant la guerre, nous n'avions pas interêt à établir une communication facile avec le Rhin superrieur parce que nous n'étions pas chez nous et que, pour essaỵer d'ouvrir la vallée clu Rhin à notre marché, nous risquions d'ouvrir on sens inverse, le Sud-Est de la France à l'importation allemande. La situation a completement changé et il est possible qu'après avoir mis le canal du Rhòne au Rhin an gabarit de la péniche on songe à le mettre au gabarit du bateau de 600 tommes. On pourrait ainsi avoir deux voies navigables à grande section réunissant le Rhòne au Rhin et contournant le Jura, l'une à l'Fst, l'autre à l'Ouest. Lacuelle de ces deux voies doit avoir pour nous la préférence? C'est une question fort complexe et qui n'est pas encore asse\% mùre pour pouvoir être résolue à l'heure actuelle.

De tout ce qui précède, il semble qu'il faut conclure que si an doit entrer résolument dans la voie de l'aménagement des forces motrices du Haut-Rhône, on doit aussi faire en sorte de ne pas s'interdire le moyen d'y établir la navigation.

\section{CONCLUSIONS}

En résumé, le programme inscrit dans la loi du 27 mai 1921 est très séduisant et il serait à désirer qu'on pût le réaliser de suite dans son intégralité. Mais si, comme cela est à craindre, il est reconnu nécessaire, pour tenir compte de certaines impossibilités praticues, de n'entreprendre cette réalisation que par étapes, il semble qu'on devrait adopter au début les directives suivantes:

Pour le Bas-Phône, maintenir et perfectionner dans la mesure du possible la navigation en laissant de côté pour le moment l'anénagement des forces motrices et s'adressant pour se procurer de l'énergie hydraulique désirable, aux affluents du Rhône.

Pour le Haut-Rhône, au contraire, entrer résolument dans la voie de l'aménagement des forces motrices mais en prenant dans la mesure du possible toutes dispositions pour que la navigation puisse être installée lorsqu'on en reconnaîtra l'utilité.

Je me rends bien compte que ces conclusions qui paraitront décevantes à beaucoup peuvent être discutées et combattues. Je voudrais seulement appeler l'attention sur le danger qu'il peut avoir à se laisser séduire par un programme théoriquement parfait, satisfaisant entièrement notre esprit de logique et de système mais destiné à rencontrer dans la pratique de grosses difficultés. N'est-il pas préférables d'envisager résolument celles-ci d'avance, plutôt que de les trouver subitement en face de soi l'heure où commencera la réalisation? Certainement ces grands programmes, que nous affectionnons en France, ont leur utilite qui est de donner à notre marche ún but précis et de nous éviter bien des erreurs et des fausses manœuvres. Mais ils ont aussi leurs dangers parce que nous sommes invinciblement portés à croire qu'étant parfaits dans la forme, ils doivent l'être aussi dans l'exécution, et alors, ou bien nous exécutons trop de travaux ou des travaux mal adaptés aux besoins qu'ils ont à satisfaire (c'est ce qui est arrivé pour le programme Freycinet), ou bien nous nous trouvons dans l'impossibilité de faire l'effort nécessaire pour réaliser un programme trop vaste, et nous ne faisons rien, ni les travaux vraiment justifiés el urgents ni les autres ; - c'est ce qui pourrait être à craindre pour le programme du Rhône.

La sagesse consisterait à choisir dans ce programme ce qui est d'une réalisation sûre, pratique et sans dangers et qui doit donner dès l'abord des avantages incontestables, et à en entrcprendre immédiatement la réalisation en soumettant le reste à un examen plus approfondi. 